\title{
Multiple processes independently predict motor learning
}

\author{
Christopher M. Perry ${ }^{1}$, Tarkeshwar Singh², Kayla G. Springer ${ }^{1}$, Adam T. Harrison ${ }^{1}$, Alexander C. McLain ${ }^{3}$ \\ and Troy M. Herter ${ }^{1 *}$ (D)
}

\begin{abstract}
Background: Our ability to acquire, refine and adapt skilled limb movements is a hallmark of human motor learning that allows us to successfully perform many daily activities. The capacity to acquire, refine and adapt other features of motor performance, such as visual search, eye-hand coordination and visuomotor decisions, may also contribute to motor learning. However, the extent to which refinements of multiple behavioral features and their underlying neural processes independently contribute to motor learning remains unknown. In the current study, we used an ethological approach to test the hypothesis that practice-related refinements of multiple behavioral features would be independently predictive of motor learning.
\end{abstract}

Methods: Eighteen healthy, young adults used an upper-limb robot with eye-tracking to practice six trials of a continuous, visuomotor task once a week for six consecutive weeks. Participants used virtual paddles to hit away 200 "Targets" and avoid hitting 100 "Distractors" that continuously moved towards them from the back of the workspace. Motor learning was inferred from trial-by-trial acquisition and week-by-week retention of improvements on two measures of task performance related to motor execution and motor inhibition. Adaptations involving underlying neural processes were inferred from trial-by-trial acquisition and week-by-week retention of refinements on measures of skilled limb movement, visual search, eye-hand coordination and visuomotor decisions. We tested our hypothesis by quantifying the extent to which refinements on measures of multiple behavioral features (predictors) were independently predictive of improvements on our two measures of task performance (outcomes) after removing all shared variance between predictors.

Results: We found that refinements on measures of skilled limb movement, visual search and eye-hand coordination were independently predictive of improvements on our measure of task performance related to motor execution. In contrast, only refinements of eye-hand coordination were independently predictive of improvements on our measure of task performance related to motor inhibition.

Conclusion: Our results provide indirect evidence that refinements involving multiple, neural processes may independently contribute to motor learning, and distinct neural processes may underlie improvements in task performance related to motor execution and motor inhibition. This also suggests that refinements involving multiple, neural processes may contribute to motor recovery after stroke, and rehabilitation interventions should be designed to produce refinements of all behavioral features that may contribute to motor recovery.

Keywords: Motor learning, Motor control, Visual search, Eye-hand coordination, Visuomotor

*Correspondence: hertert@mailbox.sc.edu

1 Department of Exercise Science, University of South Carolina, Columbia,

SC 29208, USA

Full list of author information is available at the end of the article original author(s) and the source, provide a link to the Creative Commons licence, and indicate if changes were made. The images or other third party material in this article are included in the article's Creative Commons licence, unless indicated otherwise in a credit line to the material. If material is not included in the article's Creative Commons licence and your intended use is not permitted by statutory regulation or exceeds the permitted use, you will need to obtain permission directly from the copyright holder. To view a copy of this licence, visit http://creativecommons.org/licenses/by/4.0/. The Creative Commons Public Domain Dedication waiver (http://creativeco mmons.org/publicdomain/zero/1.0/) applies to the data made available in this article, unless otherwise stated in a credit line to the data. 


\section{Introduction}

Humans learn to perform a broad repertoire of motor tasks that often require diverse and adaptable limb movements (i.e., skilled limb movements) to interact with our outside world. Many motor tasks, such as cooking, walking and driving, also employ diverse and adaptable patterns of eye movements (i.e., visual search) to actively gather visual information for planning and execution of skilled limb movements. Information gathered by visual search is also used to decide what skilled limb movements should be performed to achieve task goals (i.e., visuomotor decisions). Conversely, patterns of visual search are influenced by the available repertoire of skilled limb movements that can be used to achieve task goals. These interactions between skilled limb movements and visual search lead to coordinated patterns of eye and limb movements (e.g., eye-hand coordination). Overall, skilled limb movements, visual search, eye-hand coordination and visuomotor decisions may all contribute to learning and performance of motor tasks. However, we do not know the extent to which these behavioral features and their underlying neural processes are independently refined to produce improvements in task performance.

Given that many concepts in motor learning have unclear or ambiguous definitions, we will define several concepts based on how they are used in this study. "Motor tasks" refer to all tasks that require skilled limb movements to achieve their task goal. Accordingly, most activities of daily living (e.g., cooking, walking, driving) are considered motor tasks even if they engage perceptual, cognitive and motor functions. "Neural processes" refer to brain networks that manipulate perceptual, cognitive and motor information to perform motor tasks. "Motor learning" refers to acquisition and retention of practice-related improvements in task performance, where "task performance" refers to outcomes that are specific to achieving task goals and "improvements" necessitate increased achievement of task goals. We assume that motor learning results from neural adaptations that produce refinements of behavioral features of motor tasks (e.g., skilled limb movements, visual search, eye-hand coordination, visuomotor decisions), where "refinements" are practice-related changes that do not occur in a particular direction.

Traditional studies of motor learning have examined how skilled limb movements are refined during practice of motor tasks [1-3]. Studies of movement dynamics have found that muscle activations, joint torques and endpoint forces exhibit trial-by-trial refinements of coordination and efficiency [4-6]. Similarly, studies of movement kinematics have observed trial-by-trial refinements of speed, accuracy, smoothness and variability of skilled limb movements [7-9], and these refinements exhibit good day-by-day retention [10-13]. However, these studies were not designed to investigate if refinements of other behavioral features, such as visual search, eye-hand coordination and visuomotor decisions, contribute to motor learning.

Research on eye movements indicates that refinements of visual search may contribute to motor learning $[14,15]$. Observational studies have found that experts at different visuomotor skills have better control of eye movements than novices [16-20]. Experimental studies have also demonstrated that interventions designed to improve control of eye movements and attention lead to improvements in visuomotor performance [21-25]. While none of these studies examined trial-by-trial or week-by-week refinements of eye movements, there is ample evidence that visual search is refined during practice of perceptual tasks [26-30]. However, these studies did not examine any relationships between refinements of visual search and improvements in task performance, nor did they investigate refinements of other behavioral features. Thus, we do not know if refinements of visual search independently contribute to motor learning.

Studies of spatiotemporal coupling between eye and hand movements have provided evidence that refinements of eye-hand coordination may contribute to motor learning. Patterns of eye-hand coordination vary with task demands [31, 32] and are refined during motor learning in a task-dependent manner [33-36]. However, it remains unclear if refinements of eye-hand coordination independently contribute to improvements in task performance, or if they result from refinements of skilled limb movements and visual search but do not actually contribute to motor learning.

It is widely accepted that sensory processes contribute to planning and execution of skilled limb movements [37]. In addition, information from sensory feedback provides reinforcement that is known to play an important role in motor learning [2]. Recent studies have also found that motor learning can induce changes in visual processing that are associated with refinements of skilled limb movement $[38,39]$. This suggests that adaptations of visual and visuomotor processing contribute to motor learning. However, these studies were not designed to investigate the extent to which refinements of other behavioral features, such as visual search, eye-hand coordination and visuomotor decisions, may independently contribute to motor learning.

Despite evidence that refinements of multiple features might underlie motor learning, we do not know the extent to which they independently contribute to motor learning. Traditional experiments cannot easily address this problem because they are designed to isolate individual processes. In contrast, ethological approaches 
that study real-time, natural behavior can overcome this limitation by leveraging individual patterns of variability exhibited by several behavioral features [40]. However, this approach requires carefully controlling for any covariation between different features. For example, two or more processes may be associated with motor learning, but their individual patterns of variability might exhibit substantial covariance. This shared variance can cause regression analyses to produce incorrect estimates of the contributions made by each process. Accurate estimates of the individual contributions can only be obtained from the independent variance that remains after removing all shared variance.

The objective of the current study was to investigate the extent to which multiple neural processes might independently contribute to motor learning. Healthy young adults used an upper-limb robot with eye tracking to complete six weeks of practice of a novel, visuomotor task designed to mimic the richness of real-world visuomotor tasks. Motor learning was inferred from trial-by-trial acquisition and week-by-week retention of improvements on measures of task performance. Adaptations of multiple neural processes were inferred from trial-by-trial acquisition and week-by-week retention of refinements on measures of skilled limb movement, visual search, eye-hand coordination and visuomotor decisions. Our first hypothesis was that practicing our novel, visuomotor task would elicit trial-by-trial acquisition and week-by-week retention of improvements in task performance that are mirrored by concurrent refinements of skilled limb movements, visual search, eye-hand coordination and visuomotor decisions. Our second hypothesis was that refinements related to multiple neural processes would be independently predictive of improvements in task performance.

\section{Methods}

\section{Participants}

We recruited healthy, young adults (18-35 years old) from the University of South Carolina and surrounding areas. Participants were excluded if they reported any history of a central or peripheral neurological disorder or an ongoing musculoskeletal issue affecting either arm or hand. The study protocol was approved by the University of South Carolina's Institutional Review Board and all participants provided informed consent to participate.

\section{Apparatus}

Data were collected with a bilateral, upper-limb robot (KINARM EndPoint Lab, KINARM, Kingston, Canada) and monocular eye-tracker (EyeLink 1000, SR Research Ltd., Ottawa, Canada) that were integrated with an augmented-reality workspace (Fig. 1a) [41]. Participants sat in a custom chair that used floor-mounted tracks and hydraulics to align them with a forehead rest, which stabilized the head for eye tracking. Participants grasped two near-frictionless manipulanda, which allowed them to make two-dimensional hand movements within an $80 \mathrm{~cm}$ wide by $80 \mathrm{~cm}$ deep workspace. An opaque shield and fabric cover prevented direct vision of the hands and arms. Hand and gaze position in the robotic workspace were respectively sampled at 1000 and $500 \mathrm{~Hz}$, recorded at $200 \mathrm{~Hz}$, and filtered offline using a low-pass filter with a $20 \mathrm{~Hz}$ cutoff.

The augmented-reality environment was created in the same horizontal plane as the robotic workspace by using an inverted-monitor to project visual stimuli at $60 \mathrm{~Hz}$ through a semi-transparent mirror. Cartesian gaze position in the horizontal plane was estimated using proprietary calibration algorithms (Kinarm, Kingston, Canada) that provided accurate eye tracking within a workspace
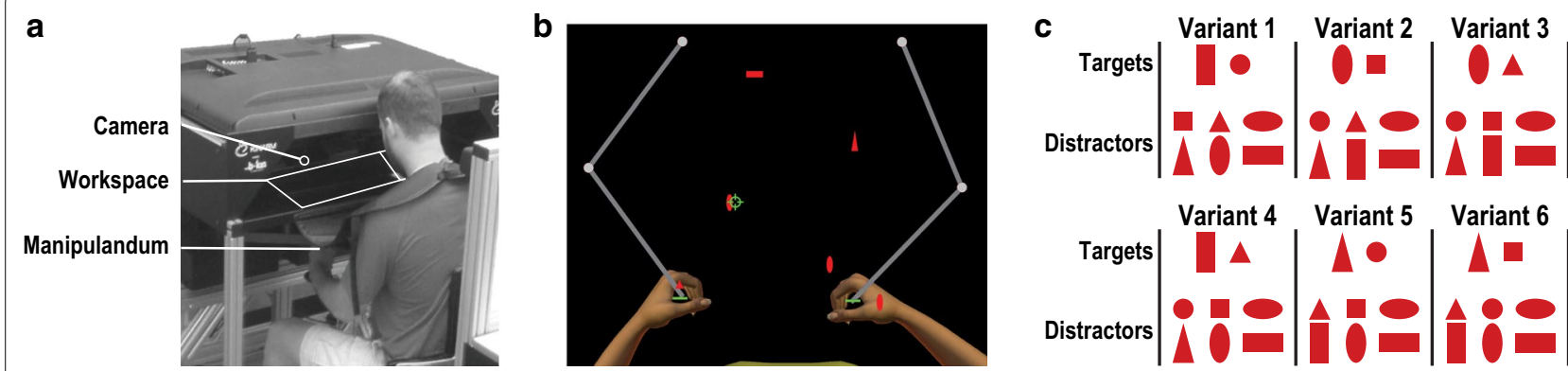

Fig. 1 Apparatus and task. a Bilateral, upper-limb robot (manipulandum), monocular eye-tracker (camera) and augmented-reality environment (workspace) used for data collection. b Overhead view of the Object Hit and Avoid (OHA) task, showing the arms and hands, robotic manipulanda, two green paddles and six red objects (geometric shapes). Participants used the two paddles to hit away 200 target objects and avoid hitting 100 distractor objects that moved toward them from the back of the workspace. The augmented-reality environment presented the paddles and objects in the same horizontal plane as the robotic workspace. Participants were unable to see their arms and hands or the robotic manipulanda. $\mathbf{c}$ The six OHA variants comprised of six combinations of target objects (one small, one large) and distractor objects (2 small, 4 large) 
of approximately $50 \mathrm{~cm}$ wide by $50 \mathrm{~cm}$ deep. All visual stimuli were presented within this portion of the robotic workspace. A nonlinear mapping corresponded to a visual area approximately $55^{\circ}$ wide by $40^{\circ}$ deep in which stimuli located closer to participants comprised larger visual angles.

\section{Task}

Participants practiced six trials of a continuous, visuomotor task, Object Hit and Avoid (OHA) [42], once a week for six consecutive weeks. Each participant was scheduled at a consistent time of day on the same weekday to avoid potential confounds caused by circadian rhythms and to assure a consistent retention interval between sessions. Illumination of the room was maintained at a constant level for the duration of the study.

In each trial of the OHA task, 300 red objects comprised of eight geometric shapes (e.g., square, circle, triangle, etc.) moved from the back of the workspace towards the participants along ten parallel paths $(5 \mathrm{~cm}$ center-to-center spacing) (Fig. 1b). Two shapes were predefined as "Targets" and six shapes were predefined as "Distractors". Each parallel path contained 20 Targets $(\mathrm{n}=200)$ and 10 Distractors $(\mathrm{n}=100)$ that were released in random order. The average number of objects that were simultaneously present in the workspace and the average speed that objects moved progressively increased over time. As a result, task difficulty increased within each trial, whereas the overall difficulty of each trial was consistent. Each trial ended after all 300 objects had passed through the workspace $(\sim 2 \mathrm{~min})$.

Participants received standardized instructions to use two green paddles $(2.5 \mathrm{~cm}$ wide) located on top of each hand to hit away as many Targets and to avoid hitting as many Distractors as possible. When participants made paddle contact with Targets, the robot applied a small perturbation (10 Newtons for $50 \mathrm{~ms}$ ) to the participant's hand and Targets rebounded from the paddle with the same direction and speed as the paddle movement. When participants made paddle contact with Distractors, no perturbation was applied to the participant's hand and Distractors passed unaltered through the paddle. Paddle size, object size and the spacing between adjacent paths prevented participants from simultaneously hitting two objects with the same hand.

We employed six distinct variants of targets and distractors to prevent overlearning of a specific variant from causing plateaus in task performance (Fig. 1c). Each variant was pseudo-randomized and counter-balanced between participants each week and was never practiced by a participant in more than one week. Specifically, each of the six variants was assigned to three different participants each week, and each participant performed six trials of a different variant each week. Before starting each trial, the two target shapes were presented in the middle of workspace until participants confirmed that they had memorized the shapes and were ready to begin. After each trial, participants were offered a rest period until they were ready to start the next trial.

\section{Gaze classification}

Gaze data were processed and classified using the procedures of a validated methodology for processing gaze data our group previously published [41]. In brief, the methodology involves preprocessing gaze data to remove blink artifacts, one sample spikes caused by incorrect corneal detection, and outliers that occurred when gaze moved outside the eye-tracking workspace. We subsequently use a novel geometric method to transform gaze position data into rotational kinematics of the eye. Finally, we use adaptive thresholding methods to classify eye movements into saccades (rapid eye movements between targets) and smooth pursuits (eye movements that followed moving targets with foveal vision). Our previous manuscript demonstrated that our methodology for gaze processing and classification correctly classifies approximately $90 \%$ of saccades and smooth pursuits and misclassifies approximately $5 \%$ of saccades and smooth pursuits when compared with manual classification (gold standard) [41].

\section{Measures}

We used hand and gaze data to compute measures of Task Performance, Skilled Limb Movement, Visual Search, EyeHand Coordination and Visuomotor Decisions for each OHA trial.

Task performance We computed two measures of task performance (Eqs. 1 and 2). Targets Hit (\%) quantified goal achievement resulting from successful execution of hand movements to hit targets (motor execution). It was calculated as the percent of all 200 targets that participants "hit", where a target was counted as "hit" if either paddle made contact with the target, causing it to move toward the back of the workspace. Only one "hit" was counted if a target was hit more than once. Distractors Avoided (\%) quantified goal achievement resulting from successful inhibition of hand movements to avoid distractors. It was calculated as the percent of all 100 Distractors that were "not hit", where a distracter was counted as "not hit" if neither paddle made contact with the distractor or if a paddle made contact but caused the distractor to move toward the front of the workspace.

$$
\text { TargetsHit }=\frac{N_{\text {TargetsHit }}}{200 \text { Targets }} * 100 \%
$$




$$
\text { DistractorsAvoided }=\frac{N_{\text {DistractorsNotHit }}}{100 \text { Distractors }} * 100 \%
$$

Skilled limb movement We computed five measures of skilled limb movement (Eqs. 3-7). Mean Hand-Speed $(\mathrm{cm} / \mathrm{s})$ quantified the overall execution speed of all hand movements by computing the average speed of right- and left-hand movements. Mean Hand-Area $\left(\mathrm{cm}^{2}\right)$ quantified the overall spatial distribution of all hand movements by calculating the average area covered by right- and left-hand movements, where each area was obtained by computing the convex hull of left- and right-hand movements. Target Contact Speed $(\mathrm{cm} / \mathrm{s})$ quantified the execution speed of skilled hand movements by computing the average speed of hand movements at the onset of paddle-contact with each target that was successfully hit. Hand-Speed Bias quantified bimanual coordination by computing inter-limb differences in movement speed. It was calculated as the normalized difference between the average speed of rightand left-hand movements. Hand-Area Bias quantified bimanual coordination by computing inter-limb differences in the spatial distributions of hand movements. It was calculated as the normalized difference between the area covered by movements of the right and left hands. Values of hand-speed bias or hand-area bias near zero indicate equal use of both hands and increasingly higher values indicate greater use of one hand than the other. We were unable to quantify many traditional measures of skilled limb movement, such as time to peak velocity, peak acceleration or smoothness, because we could not identify a distinct start or end point of most limb movements due to the continuous nature of our task.
Visual search We computed three measures of visual search (Eqs. 8-10). Objects Foveated (\%) quantified the overall efficiency of visual search by calculating the percent of all 300 objects that participants "foveated" with pursuit eye movements, where an object was counted as "foveated" if the object was followed with foveal vision for at least $40 \mathrm{~ms}$ [41]. If an object was foveated more than once, it was only counted once. Spatial Foveation Bias quantified spatial biases in the distribution of visual search by computing the normalized difference between the number of objects foveated on the right and left sides of the workspace. Extrafoveal Hits (\%) quantified covert use of parafoveal and peripheral vision for visual search by calculating the percent of targets that were hit but were not previously foveated. We were unable to compute other measures of visual search because a large number of catch-up saccades during pursuit prevented accurate calculation of other valid measures.

$$
\begin{aligned}
& \text { ObjectsFoveated }=\frac{N_{\text {ObjectsFov }}}{300 \text { Objects }} * 100 \% \\
& \text { SpatialFoveationBias }=\left|\frac{N_{\text {ObjectsFovonRight }}-N_{\text {ObjectsFovonLeft }}}{N_{\text {ObjectsFovonRight }}+N_{\text {ObjectsFovonLeft }}}\right|
\end{aligned}
$$

$$
\text { ExtrafovealHits }=\frac{N_{\text {TargetsHit } \cap \text { NotFoveated }}}{N_{\text {TargetsNotFoveated }}} * 100 \%
$$

Eye-hand coordination We computed two measures of eye-hand coordination (Eqs. 11-12). Gaze-Hand Distance $(\mathrm{cm})$ quantified spatial coupling between the eyes and

$$
\text { MeanHand }- \text { Speed }=\frac{\text { Hand }-\bar{S}_{\text {Seed }} \text { Right }+ \text { Hand }-\overline{\text { Speed }}_{\text {Left }}}{2 \text { Hands }}
$$

MeanHand - Area $=\frac{\text { Hand }- \text { Area }_{\text {Right }}+\text { Hand }- \text { Area }_{\text {Left }}}{2 \text { Hands }}$ hands by calculating the distance between gaze and hand position at the onset of paddle-contact with each target [33]. Gaze-Hand Latency (ms) quantified temporal cou-

$$
\text { Hand }- \text { SpeedBias }=\left|\frac{\text { Hand }-\bar{S}_{\text {Seed }}{ }_{\text {Right }}-\text { Hand }_{-}-\text {Speed }_{\text {Left }}}{\text { Hand }-\overline{\text { Speed }}_{\text {Right }}+\text { Hand }-\overline{\text { Speed }}_{\text {Left }}}\right|
$$

Hand - AreaBias $=\left|\frac{\text { Hand }- \text { Area }_{\text {Right }}-\text { Hand }- \text { Area }_{\text {Left }}}{\text { Hand }- \text { Area }_{\text {Right }}+\text { Hand }- \text { Area }_{\text {Left }}}\right|$

TargetContactSpeed $=\frac{\sum_{1}^{N} \text { Hand }- \text { Speed }_{\text {TargetContact }}}{N_{\text {TargetsHit }}}$ pling between eyes and hands by calculating the interval between the initial time of each target hit and final time that gaze foveated the target [33-36, 43]. If a target was hit more than once, only the first hit was included in these calculations. If a target was not foveated or was hit before it was foveated, it was excluded from these calculations. 


$$
\begin{aligned}
& \text { Gaze }- \text { HandDistance }=\frac{\sum_{1}^{N} \sqrt{\left(X_{\text {Gaze }}-X_{\text {Target }}\right)^{2}+\left(Y_{\text {Gaze }}-Y_{\text {Target }}\right)^{2}}}{N_{\text {TargetsHit }}} \\
& \text { Gaze }- \text { HandLatency }=\frac{\sum_{1}^{N}\left(\text { Time }_{\text {InitialContact }}-\text { Time }_{\text {FinalFov }}\right)}{N_{\text {TargetsHit }}}
\end{aligned}
$$

Visuomotor decisions We computed three measures of visuomotor decisions (Eqs. 13-15). Target Foveation Time (ms) quantified the amount of time used for making decisions to hit targets and was calculated as the average duration that participants foveated targets. Distractor Foveation Time $(\mathrm{ms})$ quantified the amount of time used for making decisions to avoid distractors and was calculated as the average duration that participants foveated distractors. If a target or distractor was foveated more than one time, we included the total time of all foveations. Both measures quantified the average time used to recognize and classify shapes as a target or distractor. However, Target Foveation Time included the average time used to initiate hand movements, whereas Distractor Foveation Time included the average time used to inhibit hand movements. Foveation Time Difference (ms) quantified differences between the amount of time used for making decisions to hit targets and avoid distractors and was calculated as the difference between target and distractor foveation times.

$$
\begin{aligned}
& \text { TargetFoveationTime }=\frac{\sum_{1}^{N} \text { TargetFovTime }}{N_{\text {TargetsFoveated }}} \\
& \text { DistractorFoveationTime }=\frac{\sum_{1}^{N} \text { DistractorFovTime }}{N_{\text {DistractorsFoveated }}}
\end{aligned}
$$

with the highest coefficient of variance from further analyses [44]. We subsequently performed a visual inspection of our data, which revealed the presence of a small number of outliers in several measures. For all subsequent analyses, we minimized the potential influence of outliers by performing robust regression with a Welsch weighting function [45]. Finally, we standardized each measure to obtain a mean of zero and standard deviation of one, which allowed us to compare measures with different units.

\section{Practice-related refinements}

Our first hypothesis was that practice would induce trial-by-trial and week-by-week refinements of skilled limb movement, visual search, eye-hand coordination and visuomotor decisions that mirror improvements in task performance. We tested this hypothesis by using robust regression to compare eight different linear mixed-effects models that quantified trial-by-trial acquisition and week-by-week retention of refinements (Eqs. 16-23). The first four models implemented different combinations of linear and logarithmic growth rates (linear-linear, linear-logarithmic, logarithmic-linear, logarithmic-logarithmic) to quantify trial-by-trial acquisition and week-by-week retention of refinements (Fig. 2). The other four models added an interaction term that quantified trial-by-trial changes across weeks.

FovTimeDiff $=$ TargetFoveationTime - DistractorFoveationTime

\section{Analysis}

All analyses were performed using Matlab 2017b (Mathworks Inc., Natick, MA).

\section{Validation of measures}

Since most of our measures were novel, we first examined each measure for uniqueness of information and for the presence of outliers. We confirmed that each measure quantified unique information by examining the covariance between each pair of measures. If we found a moderate Pearson correlation coefficient between any pair of measures $\left(|r| \geq 0.707, r^{2} \geq 0.5\right)$, we excluded the measure

$$
\begin{aligned}
& Y_{i j k}=b_{i}+\beta_{1} \text { Trial }_{j}+\beta_{2} \text { Week }_{k}+\epsilon_{i j k} \\
& Y_{i j k}=b_{i}+\beta_{1} \log _{\text {Trial }}+\beta_{2} \text { Week }_{\mathrm{k}}+\epsilon_{\mathrm{ijk}} \\
& Y_{i j k}=b_{i}+\beta_{1} \text { Trial }_{j}+\beta_{2} \log \text { Week }_{\mathrm{k}}+\epsilon_{\mathrm{ijk}} \\
& Y_{i j k}=b_{i}+\beta_{1} \log _{\text {Trial }}+\beta_{2} \operatorname{logWeek}_{\mathrm{k}}+\epsilon_{\mathrm{ijk}} \\
& Y_{i j k}=b_{i}+\beta_{1} \text { Trial }_{j}+\beta_{2} \text { Week }_{k}+\beta_{3}\left(18 \text { Trial }_{j} * \text { Week }_{k}\right)+\epsilon_{i j k}
\end{aligned}
$$



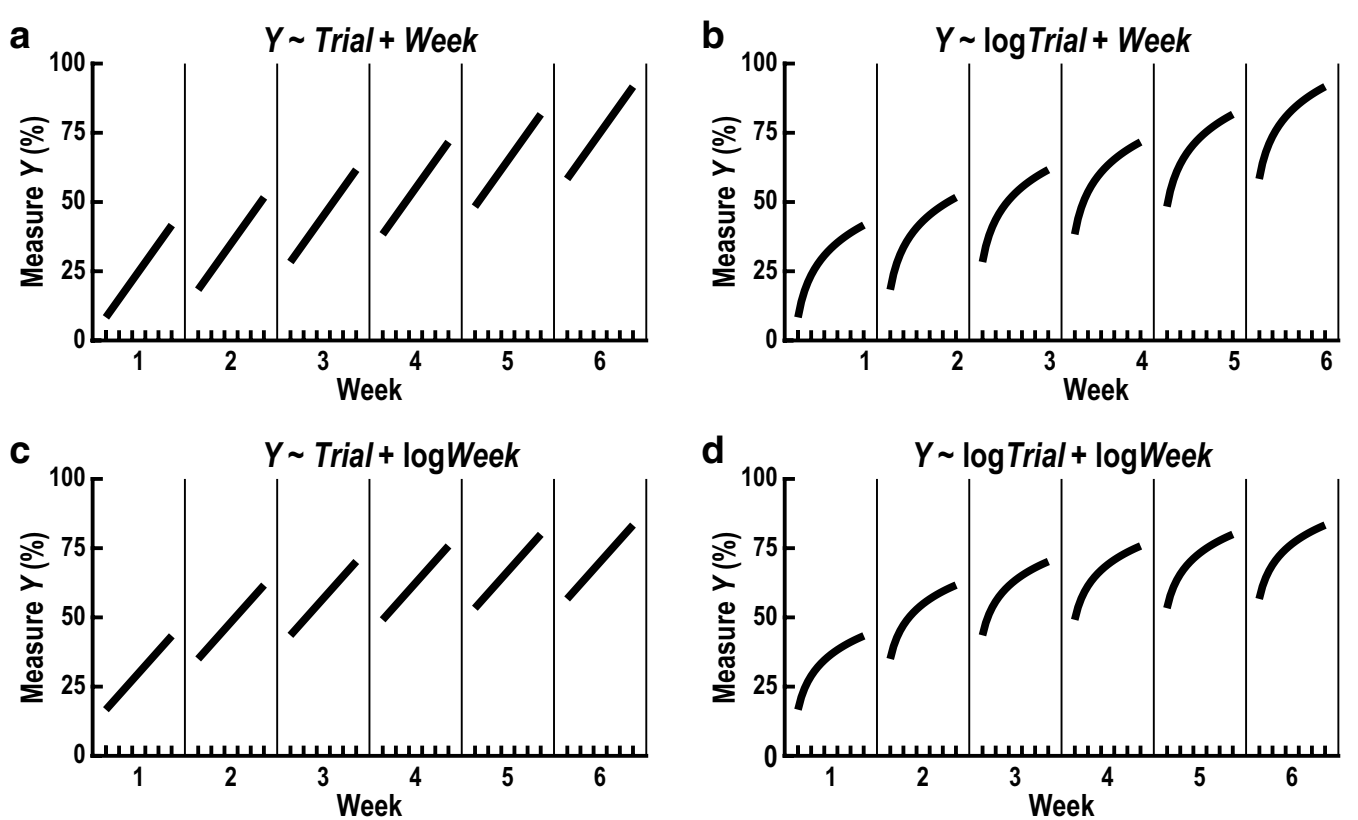

Fig. 2 Theoretical models used to quantify trial-by-trial acquisition and week-by-week retention of refinements. a Linear trial-by-trial and linear week-by-week refinements. $\mathbf{b}$ Logarithmic trial-by-trial and linear week-by-week refinements. $\mathbf{c}$ Linear trial-by-trial and logarithmic week-by-week refinements. $\mathbf{d}$ Logarithmic trial-by-trial and logarithmic week-by-week refinements

$$
\begin{aligned}
& Y_{i j k}=b_{i}+\beta_{1} \log \text { Trial }_{\mathrm{j}}+\beta_{2} \text { Week }_{\mathrm{k}}+\beta_{3}\left(\log \operatorname{Trial}_{\mathrm{j}} * \mathrm{Week}_{\mathrm{k}}\right)+\epsilon_{\mathrm{ijk}} \\
& Y_{i j k}=b_{i}+\beta_{1} \operatorname{Trial}_{j}+\beta_{2} \operatorname{logWeek}_{\mathrm{k}}+\beta_{3}\left(\text { Trial }_{\mathrm{j}} * \log _{\mathrm{Week}}\right)+\epsilon_{\mathrm{ijk}} \\
& Y_{i j k}=b_{i}+\beta_{1} \log \operatorname{Trial}_{\mathrm{j}}+\beta_{2} \operatorname{logWeek}_{\mathrm{k}}+\beta_{3}\left(\log \operatorname{Trial}_{\mathrm{j}} * \log \mathrm{Week}_{\mathrm{k}}\right)+\epsilon_{\mathrm{ijk}}
\end{aligned}
$$

In Eqs. 16-23, $Y_{i j k}$ represents each measure obtained from participant $i$, in trial $j$ of week $k, b_{i}$ is a random intercept for each participant, $\beta_{1}$ describes trial-by-trial acquisition of refinements, $\beta_{2}$ describes week-by-week retention of refinements, and $\epsilon_{i j k}$ is the error term. In Eqs. 20-23, $\beta_{3}$ is an interaction term that describes changes in trial-by-trial refinements across weeks. The model with the lowest Bayesian Information Criterion (BIC) was used to examine trial-by-trial acquisition and week-by-week retention of refinements. After finding the best-fit model for each measure, we verified that additional transformations were not required by visually inspecting the fit between the predicted and actual outcomes and by testing the residuals for normality with Kolmogorov-Smirnoff tests. Measures with at least a small effect size $\left(f^{2} \geq 0.02\right.$; [46] for trial-by-trial acquisition $\left(\beta_{1}\right)$ or week-by-week retention $\left(\beta_{2}\right)$ of refinements were subsequently included as "predictor measures" in the following analyses of our second hypothesis.

\section{Prediction of motor learning}

Our second hypothesis was that refinements related to multiple neural processes would be independently predictive of improvements in task performance. We tested this hypothesis by using multiple regression to quantify the extent to which refinements of predictor measures were independently predictive of improvements on our two measures of task performance (outcome measures). Before performing these multiple regression analyses, we first reduced the number of predictor measures included in each model by using bivariate regression to confirm that each predictor measure that was individually related to improvements on our two measures of task performance (i.e., at least a small effect size, $f^{2} \geq 0.02$ ). We then examined each predictor measure for multicollinearity by 


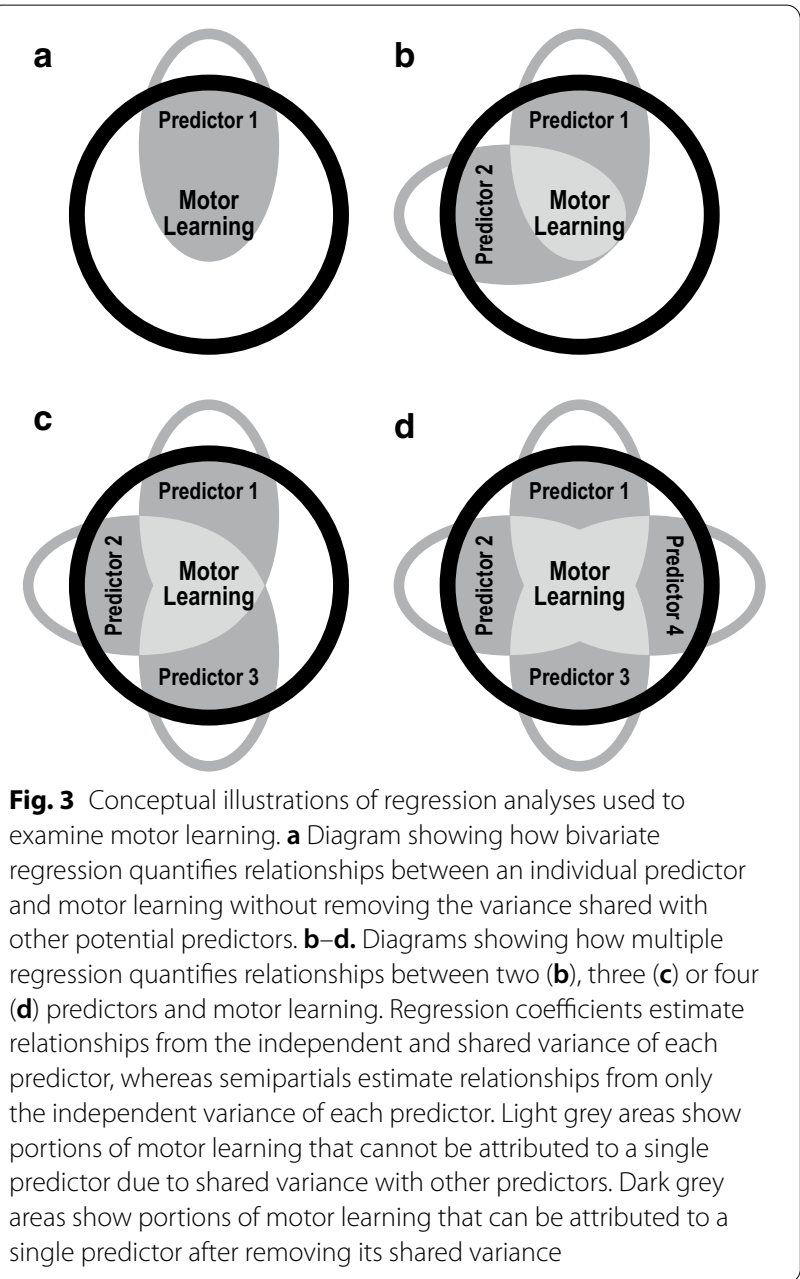

computing the Tolerance of each measure, which is the proportion of variance not explained by linear combinations of all other predictors (i.e., $1-R^{2}$ ) [47]. We subsequently performed multiple regression using linear mixed-effects models that only included the predictor measure identified in the previous step (Eq. 24).

$$
Y_{i j k}=b_{i}+\beta_{1} X_{1(i j k)}+\beta_{2} X_{2(i j k)}+\cdots+\beta_{N} X_{n(i j k)}+\epsilon_{i j k}
$$

In Eq. 24, $Y_{i j k}$ represents task performance of participant $i$ in trial $j$ of week $k, b_{i}$ are random intercepts for each participant, coefficients $\beta_{1}-\beta_{N}$ are estimated relationships between each predictor measure $\left(X_{1}-X_{n}\right)$ and the respective measure of task performance, and $\epsilon_{i j k}$ is the error term.

We finally identified each predictor measure that was independently predictive of improvements on our two measures of task performance. Importantly, the values of coefficients $\beta_{1}-\beta_{N}$ in Eq. 24 are influenced by variance that is independent of all other predictors and variance that is shared with other predictors. Figure 3 illustrates conceptual representations of independent and shared variance for four theoretical regression models that include one, two, three, or four predictors of motor learning. If only one predictor is examined (a), it might be assumed that all variance related to motor learning (dark grey area) is independently predictive of motor learning. However, if multiple predictors are examined (b-d), part of each predictor's variance related to motor learning would be independent of all other predictors (dark grey area) and part would be shared with other predictors (light grey area). The relationships between each predictor's independent variance and motor learning are described by semipartial coefficients of determination $\left(s r^{2}\right)$. For the purpose of our second hypothesis, we calculated semipartial coefficients of determination $\left(s r^{2}\right)$, semipartial effect sizes $\left(s f^{2}\right)$, and semipartial $p$-values $(s p)$ to examine the relationships between the independent variance of each predictor measure and improvements on our two measures of task performance. We considered measures with at least a small semipartial effect size $\left(s f^{2} \geq 0.02\right)$ as meaningful predictors of motor learning, though we recognize that this could underestimate the amount of motor learning that should be attributed to each predictor.

For the purpose of rigor and reproducibility, we validated our multiple regression results by performing forward and backward stepwise regression with the same set of predictor measures used in our multiple regression analyses. We used the BIC to determine which predictor to add or remove at each step. This resulted in a final model with a minimum BIC.

\section{Results}

\section{Participants}

We enrolled 18 healthy, young adults (8 male, 10 female; $24.2 \pm 3.7$ years old; 17 R-handed, 1 L-handed) in the study. One participant was unable to complete the sixth week of the study. We included the participant's data without replacement of the sixth week.

\section{Exemplar OHA performance}

Figure 4 illustrates pursuit and saccadic eye movements (pink and gold lines) and left- and right-hand movements (blue and red lines) made by an exemplar participant at four time points, Week $1 \cdot \operatorname{Trial}_{1}(\mathrm{a}), \mathrm{Week}_{1} \cdot \mathrm{Trial}_{6}$ (b), Week $_{6} \cdot \operatorname{Trial}_{1}$ (c), and Week $_{6} \cdot \operatorname{Trial}_{6}$ (d). At each time point, the participant's eye movements covered an area of approximately $50 \mathrm{~cm}$ wide $(X)$ by $40 \mathrm{~cm}$ deep $(Y)$. The center-of-mass was consistently located near the midline but shifted distally from around $30 \mathrm{~cm}$ on Week $1 \cdot \operatorname{Trial}_{1}(\mathrm{a})$, to $35 \mathrm{~cm}$ on $\mathrm{Week}_{1} \cdot \operatorname{Trial}_{6}(\mathrm{~b})$, and $40 \mathrm{~cm}$ on Week $_{6} \cdot$ Trial $_{1}$ and Week $_{6} \cdot \operatorname{Trial}_{6}$ (c, d). Combined 

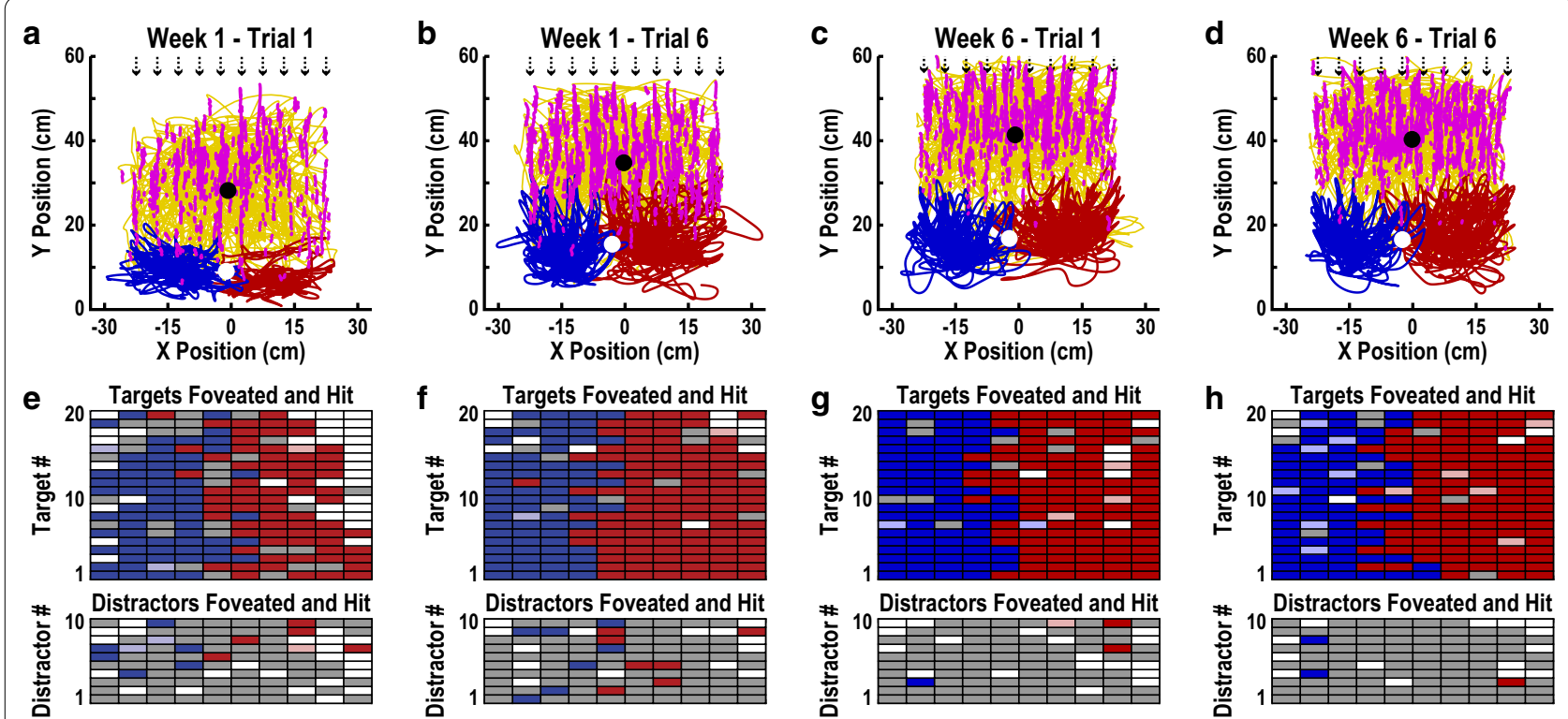

Fig. 4 Eye and hand movements and target/distractor foveations and hits by an exemplar participant during two trials on Week 1 and two trials on Week 6. a-d. X position (width) versus Y position (depth) of eye and hand movements on Week Trial $_{1}(\mathbf{a})$, Week $_{1} \cdot \operatorname{Trial}_{6}(\mathbf{b})$, Week Wrial $_{1}(\mathbf{c})$, and Week $_{6} \cdot \operatorname{Trial}_{6}(\mathbf{d})$. Colored lines illustrate pursuit eye movements (pink), saccadic eye movements (gold), left-hand movements (blue) and right-hand movements (red). Dashed arrows indicate the ten parallel paths that objects moved along. Black and white circles show the Center-of-Mass of gaze

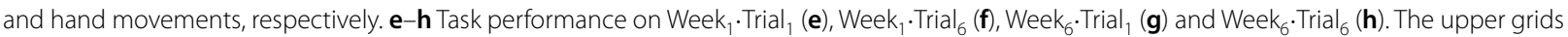
$(20 \times 10)$ represent each target and the lower grids $(10 \times 10)$ represent each distractor that was foveated and hit with the left hand (dark blue), foveated and hit with the right hand (dark red), foveated but not hit (grey), not foveated but hit with the left hand (light blue), not foveated but hit with the right hand (light red), or neither foveated nor hit (white)

movements of both hands covered an area that was around $50 \mathrm{~cm}$ wide and consistently centered near the midline. However, the range of hand movements in depth increased from around $15 \mathrm{~cm}$ on $\mathrm{Week}_{1} \cdot \operatorname{Trial}_{1}$ (a) to $20 \mathrm{~cm}$ on the other three trials (b-d). The center-of-mass also shifted distally from under $10 \mathrm{~cm}$ on $\mathrm{Week}_{1} \cdot \operatorname{Trial}_{1}$ (a) to over $15 \mathrm{~cm}$ on the other three trials (b-d). Left- and right-hand movements covered similar areas and were largely constrained to their respective sides.

Figure 4 also displays grids of rectangles that represent each Target (upper grids: $20 \times 10$ ) and Distractor (lower grids: $10 \times 10$ ) that was foveated and hit (left hand: dark blue, right hand: dark red), foveated but not hit (grey), not foveated but hit (left hand: light blue, right hand: light red), or neither foveated nor hit (white). The participant failed to foveate several targets and distractors on $\mathrm{Week}_{1} \cdot \operatorname{Trial}_{1}$ (e) but foveated the majority of targets and distractors on the other three trials $(f-h)$. Similarly, the participant failed hit a number of targets on $\mathrm{Week}_{1} \cdot$ Trial $_{1}$ (e) but hit the majority of targets on the other three trials (f-h). In contrast, the participant hit several distractors in the first week (e, f) but very few in the last week (f-h). At all four time points, the participant hit more targets with the right-hand, including several targets on the left side of the workspace.

\section{Validation of measures}

Targets Hit and Distractors Avoided exhibited a low correlation $(r=0.03)$, indicating that they quantified unique aspects of task performance. Both measures were included in our subsequent analyses. We also examined each pair of predictor measures for high correlations $(|r|$ $\geq 0.707$ ) indicative of redundant information (Table 1 ). Two pairs exhibited high correlations, Mean Hand-Speed and Target Contact Speed $(r=0.89)$ and Gaze-Hand Distance and Gaze-Hand Latency $(r=0.89)$. Target Contact Speed and Gaze-Hand Latency were excluded from all remaining analyses because they had the highest coefficients of variance in each pair [44].

\section{Confirmation of motor learning}

Before testing our two hypotheses, we first confirmed that our participants demonstrated trial-by-trial acquisition and week-by-week retention of improvements in task performance (Table 2). Targets Hit exhibited moderate trial-by-trial increases $\left(\beta_{1}=0.26, f^{2}=0.23, p<10^{-6}\right)$, large week-by-week increases $\left(\beta_{2}=0.49, f^{2}=0.82, p\right.$ $\left.<10^{-6}\right)$, and small trial-by-trial decreases across weeks $\left(\beta_{3}=-0.16, f^{2}=0.09, p<10^{-6}\right)$ (Fig. 5a). We also observed small, week-by-week increases on Distractors Avoided $\left(\beta_{2}=0.20, f^{2}=0.11, p<10^{-6}\right)$ (Fig. 5b). These finding show 


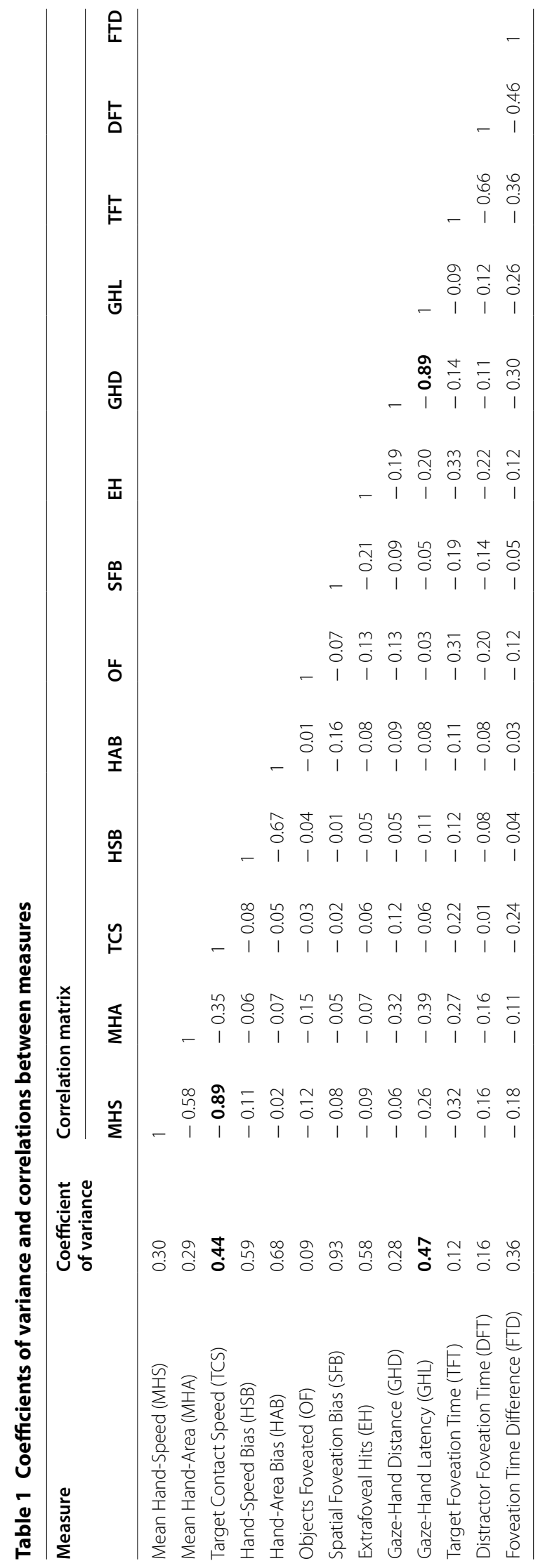




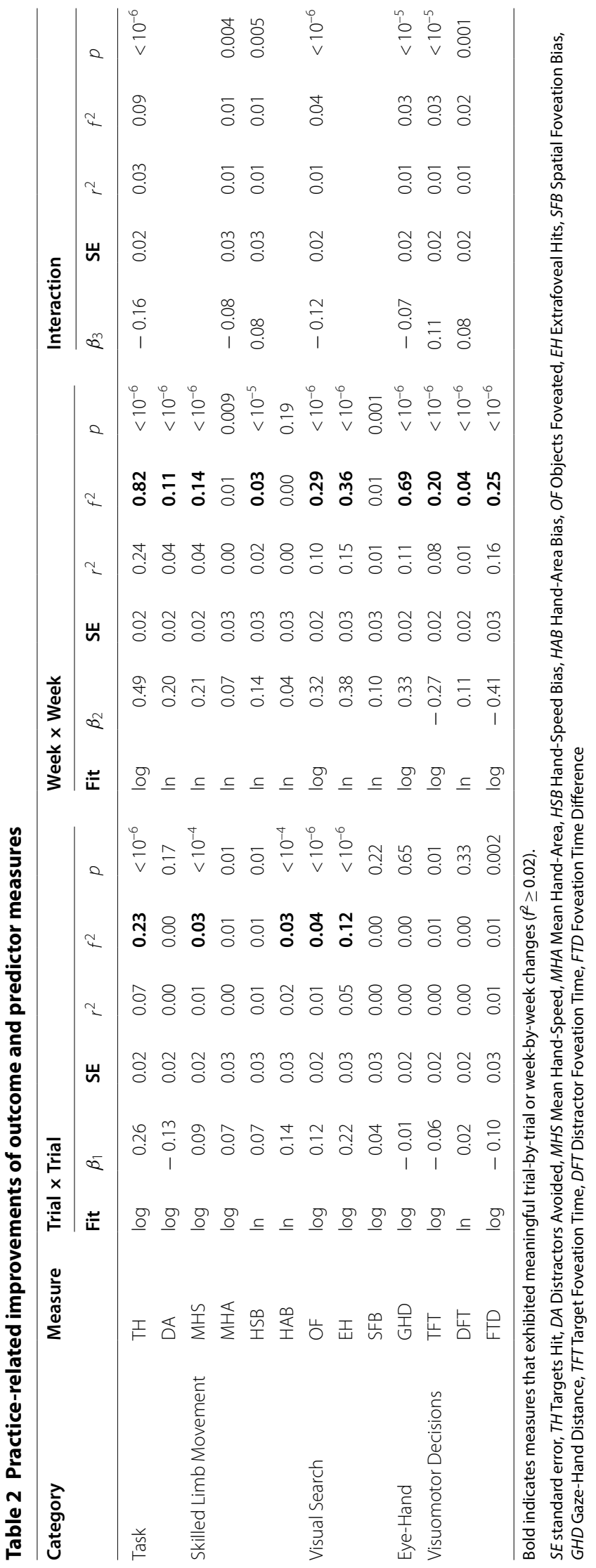



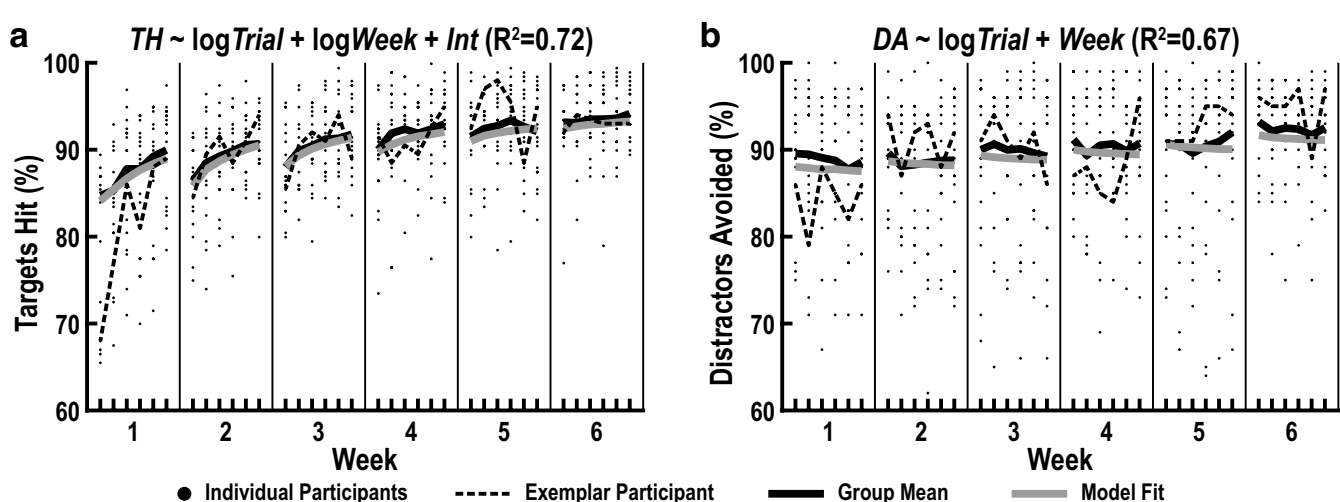

Fig. 5 Improvements on measures of task performance. Trial-by-trial acquisition and week-by-week retention of improvements on Targets Hit (a) and Distractors Avoided (b). Each panel shows raw data values of all individual participants (small black dots), raw data values of the exemplar participant in Fig. 4 (thin dashed lines), group means of the reweighted participant data (thick black lines), and model predictions (thick grey lines) Reweighted participant data were obtained by applying weights from the robust regression to the raw data values of all participants. The model with the best overall fit (lowest Bayesian Information Criterion) is displayed at the top of each panel
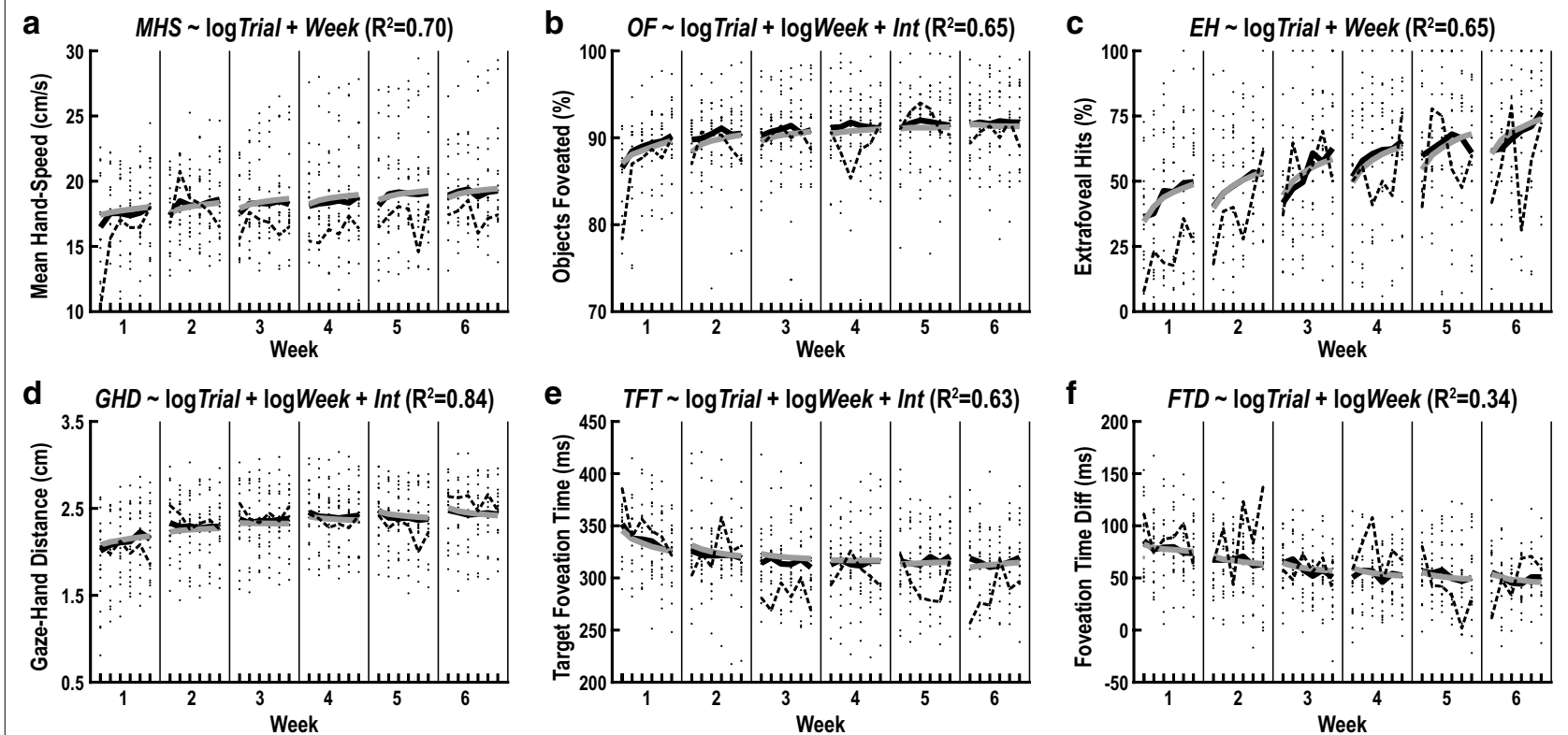

Fig. 6 Refinements on measures of behavioral features. Trial-by-trial acquisition and week-by-week retention of refinements on Mean Hand-Speed (a), Objects Foveated (b), Extrafoveal Hits (c), Gaze-Hand Distance (d), Target Foveation Time (e) and Foveation Time Difference (f). Each panel shows raw data values of all individual participants (small black dots), raw data values of the exemplar participant in Fig. 4 (thin dashed lines), group means of the reweighted participant data (thick black lines), and model predictions (thick grey lines). Reweighted participant data were obtained by applying weights from the robust regression to the raw data values of all participants. The model with the best overall fit (lowest Bayesian Information Criterion) is displayed at the top of each panel

that practice-related improvements in motor execution (Targets Hit) and motor inhibition (Distractors Avoided) contributed to improvements in task performance.

\section{Practice-related refinements}

We tested our first hypothesis by examining trialby-trial acquisition and week-by-week retention of refinements on our measures of skilled limb movement, visual search, eye-hand coordination and visuomotor decisions (Table 2). Three measures of skilled limb movement (Mean Hand-Speed, Hand-Speed Bias, and Hand-Area Bias) displayed practice-related refinements. Mean Hand-Speed exhibited small trial-by-trial increases $\left(\beta_{1}=0.09, f^{2}=0.03, p<10^{-4}\right)$ and small week-by-week 
increases $\left(\beta_{2}=0.21, f^{2}=0.14, p<10^{-6}\right)$ (Fig. 6a). HandSpeed Bias demonstrated small week-by-week increases $\left(\beta_{2}=0.14, f^{2}=0.03, p<10^{-5}\right)$ and Hand-Area Bias showed small trial-by-trial increases $\left(\beta_{1}=0.14, f^{2}=0.03, p<10^{-4}\right)$. Two measures of visual search (Objects Foveated and Extrafoveal Hits) exhibited practice-related refinements. Objects Foveated displayed small trial-by-trial increases $\left(\beta_{1}=0.12, f^{2}=0.04, \quad p<10^{-6}\right)$, moderate week-by-week increases $\left(\beta_{2}=0.32, f^{2}=0.29, p<10^{-6}\right)$, and small trial-bytrial decreases across weeks $\left(\beta_{3}=-0.12, f^{2}=0.04, p<10^{-}\right.$ ${ }^{6}$ ) (Fig. 6b). Extrafoveal Hits exhibited small trial-by-trial increases $\left(\beta_{1}=0.22, f^{2}=0.12, p<10^{-6}\right)$ and large weekby-week increases $\left(\beta_{2}=0.38, f^{2}=0.36, p<10^{-6}\right)$ (Fig. 6c). Our only measure of eye-hand coordination, Gaze-Hand Distance, demonstrated large week-by-week increases $\left(\beta_{2}\right.$ $\left.=0.33, f^{2}=0.69, p<10^{-6}\right)$ (Fig. $6 \mathrm{~d}$ ). All three measures of visuomotor decisions (Target Foveation Time, Distractor Foveation Time and Foveation Time Difference) displayed practice-related refinements. Target Foveation Time showed moderate week-by-week decreases $\left(\beta_{2}\right.$ $=-0.27, f^{2}=0.20, p<10^{-6}$ ) (Fig. 6e). Distractor Foveation Time displayed small week-by-week increases $\left(\beta_{2}=0.11\right.$, $\left.f^{2}=0.04, p<10^{-6}\right)$. Foveation Time Difference exhibited moderate week-by-week decreases $\left(\beta_{2}=-0.41, f^{2}=0.25\right.$, $p<10^{-6}$ ) (Fig. 6f). One measure of skilled limb movement (Mean Hand-Area) and one measure of visual search (Spatial Foveation Bias) did not exhibit practice-related refinements and were excluded from further analyses.

\section{Prediction of motor learning}

We initially used bivariate regression to identify predictor measures that were individually related to improvements on our two measures of task performance (i.e., at least a small effect size, $f^{2} \geq 0.02$ ) (Table 3 ). We identified six predictor measures that were individually related to improvements in Targets Hit. They included Extrafoveal Hits $\left(\beta=0.70, f^{2}=1.52, p<10^{-6}\right)$ (Fig. $\left.7 \mathrm{a}\right)$, Objects Foveated $\left(\beta=0.59, f^{2}=0.80, p<10^{-6}\right)$ (Fig. $\left.7 \mathrm{~b}\right)$, Gaze-Hand Distance $\left(\beta=0.58, f^{2}=0.65, \quad p<10^{-6}\right)$ (Fig. 7c), Mean Hand-Speed $\left(\beta=0.50, f^{2}=0.48, p<10^{-6}\right)$ (Fig. $\left.7 \mathrm{~d}\right)$, Target Foveation Time $\left(\beta=-0.46, f^{2}=0.41, p<10^{-6}\right)$, and Foveation Time Difference $\left(\beta=0.23, f^{2}=0.09, p<10^{-6}\right)$. We also identified six predictor measures that were individually related to improvements in Distractors Avoided. They included Gaze-Hand Distance $\left(\beta=0.29, f^{2}=0.25, p<10^{-}\right.$ $\left.{ }^{6}\right)$, Target Foveation Time $\left(\beta=-0.11, f^{2}=0.04, p<10^{-3}\right)$, Hand-Speed Bias $\left(\beta=0.11, f^{2}=0.03, p<10^{-3}\right)$, Extrafoveal Hits $\left(\beta=0.11, f^{2}=0.03, p<10^{-3}\right)$, Foveation Time Difference $\left(\beta=-0.09, f^{2}=0.02, p<10^{-3}\right)$, and Objects Foveated $\left(\beta=0.09, f^{2}=0.02, p<0.01\right)$.

We subsequently tested our second hypothesis by using multiple regression to analyze the extent to which refinements on the preceding predictor measures were independently predictive of improvements in Target Hits and Distractors Avoided (i.e., at least a small semipartial effect size, $f^{2} \geq 0.02$ ) (Table 4). Our multiple regression identified two measures of visual search (Extrafoveal Hits: $\beta=0.54, f^{2}=0.61, s p<10^{-6}$; Objects Foveated: $\left.\beta=0.32, f^{2}=0.16, s p<10^{-6}\right)$, one measure of

Table 3 Bivariate regression between predictor and outcome measures

\begin{tabular}{|c|c|c|c|c|c|c|c|}
\hline Outcome measures & Predictor measures & $\beta$ & SE & $r^{2}$ & $f^{2}$ & $p$ & $P_{\text {(residuals) }}$ \\
\hline \multirow[t]{9}{*}{ Targets Hit } & Extrafoveal Hits & -0.70 & 0.03 & $<0.49$ & $<1.52$ & $<10^{-6}$ & 0.07 \\
\hline & Objects Foveated & -0.59 & 0.04 & $<0.35$ & $<0.80$ & $<10^{-6}$ & 0.27 \\
\hline & Gaze-Hand Distance & -0.58 & 0.05 & $<0.33$ & $<0.65$ & $<10^{-6}$ & 0.31 \\
\hline & Mean Hand-Speed & -0.50 & 0.05 & $<0.25$ & $<0.48$ & $<10^{-6}$ & 0.07 \\
\hline & Target Foveation Time & -0.46 & 0.04 & $<0.21$ & $<0.41$ & $<10^{-6}$ & 0.14 \\
\hline & Foveation Time Difference & -0.23 & 0.03 & $<0.05$ & $<0.09$ & $<10^{-6}$ & 0.05 \\
\hline & Hand-Speed Bias & -0.10 & 0.04 & $<0.01$ & $<0.02$ & 0.01 & 0.04 \\
\hline & Distractor Foveation Time & -0.06 & 0.05 & $<0.01$ & $<0.01$ & 0.23 & 0.07 \\
\hline & Hand-Area Bias & -0.02 & 0.04 & $<0.01$ & $<0.01$ & 0.65 & 0.10 \\
\hline \multirow[t]{9}{*}{ Distractors Avoided } & Gaze-Hand Distance & 0.29 & 0.04 & $<0.09$ & $<0.25$ & $<10^{-6}$ & 0.65 \\
\hline & Target Foveation Time & -0.11 & 0.04 & $<0.01$ & $<0.04$ & $<10^{-3}$ & 0.23 \\
\hline & Hand-Speed Bias & 0.11 & 0.03 & $<0.01$ & $<0.03$ & $<10^{-3}$ & 0.51 \\
\hline & Extrafoveal Hits & 0.11 & 0.03 & $<0.01$ & $<0.03$ & $<10^{-3}$ & 0.37 \\
\hline & Foveation Time Difference & -0.09 & 0.03 & $<0.01$ & $<0.02$ & $<10^{-3}$ & 0.31 \\
\hline & Objects Foveated & 0.09 & 0.04 & $<0.01$ & $<0.02$ & $<0.01$ & 0.21 \\
\hline & Distractor Foveation Time & 0.05 & 0.04 & $<0.01$ & $<0.01$ & 0.19 & 0.32 \\
\hline & Hand-Area Bias & 0.02 & 0.03 & $<0.01$ & $<0.01$ & 0.54 & 0.25 \\
\hline & Mean Hand-Speed & 0.01 & 0.04 & $<0.01$ & $<0.01$ & 0.92 & 0.14 \\
\hline
\end{tabular}



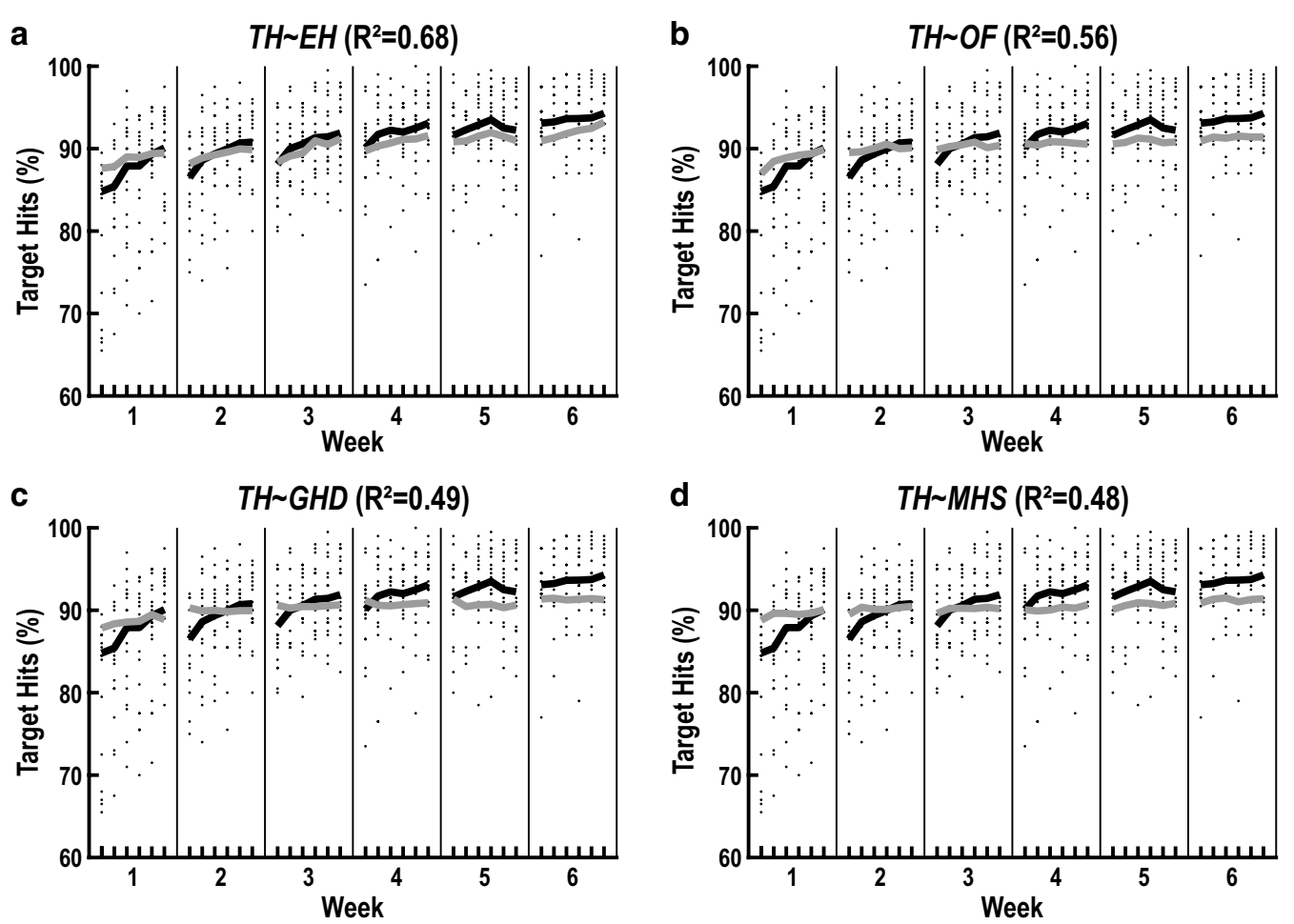

Fig. 7 Univariate predictions of task performance. Illustrations show the predicted improvements in Targets Hit obtained from bivariate regression models using Extrafoveal Hits (a), Objects Foveated (b), Gaze-Hand Distance (c) and Mean Hand-Speed (d) as predictors. Each panel shows raw data values of all individual participants (small black dots), group means of the reweighted participant data (thick black lines), and model predictions (thick grey lines). Reweighted participant data were obtained by applying weights from the robust regression to the raw data values of all participants

Table 4 Multiple regression between predictor and outcome measures

\begin{tabular}{|c|c|c|c|c|c|c|}
\hline Outcome & & $D F$ & $R^{2}$ & $F^{2}$ & $P$ & $P_{\text {(residuals) }}$ \\
\hline \multirow[t]{8}{*}{ Target Hits } & Full Model & 631 & 0.78 & 3.59 & $<10^{-6}$ & 0.71 \\
\hline & Predictors & $\beta$ & $s r^{2}$ & $s f^{2}$ & $s p$ & Tolerance \\
\hline & Extrafoveal Hits & -0.54 & 0.133 & 0.61 & $<10^{-6}$ & 0.83 \\
\hline & Objects Foveated & -0.32 & 0.034 & 0.16 & $<10^{-6}$ & 0.88 \\
\hline & Gaze-Hand Distance & -0.22 & 0.015 & 0.07 & $<10^{-3}$ & 0.83 \\
\hline & Mean Hand-Speed & -0.14 & 0.006 & 0.03 & 0.02 & 0.89 \\
\hline & Target Foveation Time & -0.11 & 0.003 & 0.01 & 0.12 & 0.72 \\
\hline & Foveation Time Difference & -0.07 & 0.002 & 0.01 & 0.08 & 0.79 \\
\hline Outcome & Predictors & $D F$ & $R^{2}$ & $F^{2}$ & $P$ & $P_{\text {(residuals) }}$ \\
\hline \multirow[t]{8}{*}{ Distractors Avoided } & Full Model & 631 & 0.66 & 1.90 & $<10^{-6}$ & 0.48 \\
\hline & Predictors & $\beta$ & $s r^{2}$ & $s f^{2}$ & $s p$ & Tolerance \\
\hline & Gaze-Hand Distance & -0.24 & 0.014 & 0.04 & 0.01 & 0.86 \\
\hline & Hand-Speed Bias & -0.09 & 0.005 & 0.01 & 0.03 & 0.95 \\
\hline & Extrafoveal Hits & -0.04 & 0.001 & 0.00 & 0.32 & 0.83 \\
\hline & Foveation Time Difference & -0.04 & 0.001 & 0.00 & 0.40 & 0.90 \\
\hline & Target Foveation Time & -0.01 & 0.000 & 0.00 & 0.94 & 0.74 \\
\hline & Objects Foveated & -0.01 & 0.000 & 0.00 & 0.95 & 0.88 \\
\hline
\end{tabular}



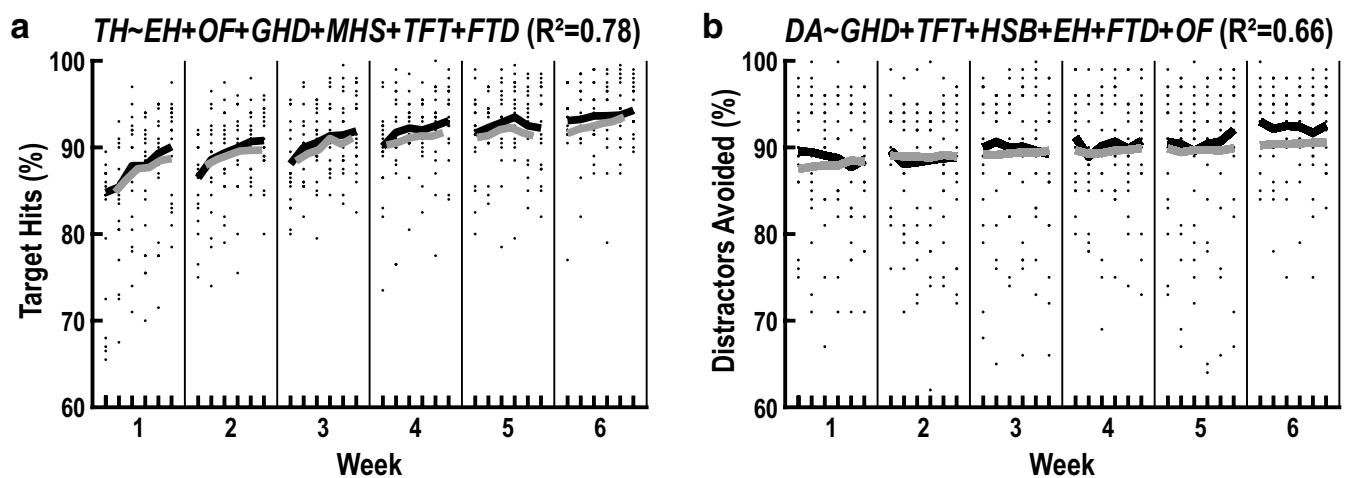

Fig. 8 Multivariate predictions of task performance. Illustrations show the predicted improvements in Targets Hit (a) and Distractors Avoided (b) obtained from multiple regression. Each panel shows raw data values of all individual participants (small black dots), group means of the reweighted participant data (thick black lines), and model predictions (thick grey lines). Reweighted participant data were obtained by applying weights from the robust regression to the raw data values of all participants

eye-hand coordination (Gaze-Hand Distance: $\beta=0.22$, $\left.f^{2}=0.07, s p<10^{-3}\right)$, and one measure of skilled limb movement (Mean Hand-Speed: $\beta=0.14, f^{2}=0.03$, sp $=0.02)$ that were independently predictive of improvements in Target Hits (Fig. 8a). In contrast, our multiple regression only identified a single measure of eye-hand coordination (Gaze-Hand Distance: $\left(\beta=0.24, f^{2}=0.04\right.$, $s p=0.01$ ) that was independently predictive of improvements on Distractors Avoided (Fig. 8b).

Finally, our stepwise regression analyses confirmed the results obtained from our multiple regression analyses. Specifically, the final model for Targets Hit only included the same measures of visual search (Extrafoveal Hits, Objects Foveated), eye-hand coordination (Gaze-Hand Distance) and skilled limb movement (Mean Hand-Speed). Furthermore, the final model for Distractors Avoided only included Gaze-Hand Distance.

\section{Discussion}

\section{Multiple processes independently predict motor learning}

The results of this study provide indirect evidence that practice-related refinements involving multiple neural processes may contribute to motor learning. Notably, we observed that measures of skilled limb movement, visual search and eye-hand coordination underwent practice-related refinements (Hypothesis 1) that were independently predictive of improvements in task performance (Hypothesis 2). Importantly, in drawing this conclusion, we assume that the trial-by-trial and weekby-week refinements exhibited by measures of skilled limb movement, visual search and eye-hand coordination can be used to infer that practice produced refinements involving multiple neural processes. Furthermore, we assume that motor learning could be inferred from trial-by-trial and week-by-week improvements exhibited by measures of task performance.

Other studies have provided evidence that both sensory and motor processes contribute to motor learning [48], but these studies were not designed to investigate the extent to which these processes are independent predictors of motor learning. As result, we do not know the extent to which relationships with motor learning reflected independent or shared variance. In the current study, we addressed the issue of covariation by examining independent predictions of motor learning after removing all shared variance. This analysis showed that skilled limb movements, visual search and eye-hand coordination are independent predictors of motor learning, indicating that studies of motor learning should account for the various processes that may influence improvements in task performance.

\section{Skilled limb movements independently predict motor learning}

Increases in Mean Hand-Speed were associated with increases in Targets Hit, indicating that participants learned to hit more targets by quickly moving their hands to different areas of the workspace. Although faster movements are more variable and less accurate [2, 9], any decreases in movement accuracy were not associated with increases in the proportion of hand movements that failed to make paddle-contact with targets. Alternatively, it is possible that optimization of intermuscular coordination may have allowed participants to move faster without incurring greater movement variability. In either 
case, increases in movement speed had a positive effect on task performance, thus our results are consistent with the principles of optimal feedback control $[49,50]$.

\section{Visual search independently predicts motor learning}

Increases in Extrafoveal Hits and Objects Foveated were the strongest independent predictors of increases in Targets Hits. These findings indicate that refinements of visual search led to better task performance by optimizing how participants gathered information with foveal and extrafoveal vision. This is consistent with evidence that visual search is highly adaptive to different task demands and environments, such as environments in which taskrelevant objects are more likely to appear at certain locations $[51,52]$.

The association between Extrafoveal Hits and Target Hits indicates that participants learned to use extrafoveal information to guide hand movements used to hit targets. This is consistent with a previous study of visual search, which found that practice led to improvements in using extrafoveal vision to search for objects with taskrelevant features [53]. In addition, cortical areas known to process peripheral visual information exhibit greater involvement during motor tasks [54]. However, to our knowledge, our study is the first to show that refinements of extrafoveal visual processing are predictive of motor learning.

The association between Targets Foveated and Target Hits suggests that refinements used to maximize the number of objects that participants foveated with visual search led to improvements in hitting targets. The modest correlation between Objects Foveated and Target Foveation Time ( $r=-0.31$; Table 1$)$ also indicates that, at least in part, decreases in the time spent foveating targets freed up time to foveate more objects. In contrast, studies of "quiet eye" have found that experts at motor tasks have longer foveations on task-relevant objects than novices $[17,19,20]$. Furthermore, training interventions designed to increase foveation durations have produced improvements in motor performance [22-25]. These divergent findings suggest that both increases and decreases in foveation times can benefit motor performance, depending on the task demands and environment. As a result, we predict that practice will lead to increases in target foveation times in tasks with high demands on accuracy and low demands on speed of visual processing, whereas practice will produce decreases in foveation times in tasks with low demands on accuracy and high demands on speed of visual processing.

\section{Eye-hand coordination independently predicts motor learning}

Increases in Gaze-Hand Distance were associated with increases in Targets Hits, indicating that looking away from targets before hitting them led to improvements in task performance. Although this contrasts with studies showing rigid coupling between initiation of eye movements and completion of hand movements [43], other studies have found that this rigid coupling decreases with practice [33-36]. We believe that increases in Gaze-Hand Distance may reflect a transition from an early reliance on visual feedback for accurate execution of hand movements to a subsequent reliance on kinesthetic feedback for accurate execution of hand movements. This would have allowed visual search to gather task-relevant information with greater efficiency [33]. Specifically, looking away from targets before hitting them would have disrupted visual feedback used to accurately guide hand movements toward targets. However, it would have enabled earlier and longer foveations of objects, thereby facilitating more efficient decisions whether to hit or avoid objects by either executing or inhibiting skilled limb movements. Importantly, any negative effects resulting from disruption of visual feedback of hand movements could be offset by a greater reliance on kinesthetic feedback, which is known to improve during motor learning [55-57] and may directly contribute to motor learning [58-61].

\section{Distinct predictors of motor execution and inhibition}

We found that motor execution (Targets Hit) and motor inhibition (Distractors Avoided) exhibited distinct patterns of improvements. Notably, Targets Hit showed trial-by-trial and week-by-week improvements, whereas Distractors Avoided displayed only week-by-week improvements. We also found that different processes were independently predictive of improvements in motor execution and inhibition. Refinements of skilled limb movements (Mean Hand-Speed), visual search (Objects Foveated, Extrafoveal Hits) and eye-hand coordination (Gaze-Hand Distance) were independently predictive of improvements in Targets Hit. In contrast, eye-hand coordination (Gaze-Hand Distance) was the only independent predictor of improvements in Distractors Avoided. Given that avoiding distractors mainly involved inhibition rather than execution of hand movements, it is not surprising that increases in Mean Hand-Speed were not predictive of increases in Distractors Avoided. In contrast, increased Gaze-Hand Distance would have facilitated both motor execution and inhibition by allowing participants more time to make decisions whether to initiate or inhibit movements. It is perhaps surprising that increases in Objects Foveated were not predictive of increases in 
Distractors Avoided. We would expect that more efficient visual search should lead to improvements in both motor execution and inhibition by allowing more objects to be processed with foveal vision. The lack of a relationship may reflect that participants exhibited smaller improvements on Distractors Avoided. However, if the proportion of targets and distractors was equal or reversed, we expect that our participants may have shown greater improvements on Distractors Avoided and we may have found a meaningful relationship.

\section{Limitations}

By examining patterns of variability exhibited by measures related to multiple neural processes, we found that refinements of multiple processes were independently predictive of motor learning. However, our paradigm and analyses were not designed to make causal inferences. This requires measuring motor learning while experimentally manipulating one process and controlling for interactions with all other processes. For example, masking objects that are not located within foveal vision would neutralize the contributions of extrafoveal hits on motor learning. If this reduced motor learning without affecting refinements of other processes, it would show that refinements of extrafoveal processing are causally linked to motor learning.

Another limitation of the current study is that we did not examine practice-related refinements of proprioception. This is an important limitation because improvements in planning and executing skilled limb movements may involve refinements that alter the processing of proprioceptive feedback [37]. In agreement with this hypothesis, previous studies have demonstrated that motor learning is associated with modifications of rapid responses to proprioceptive feedback [62] and improvements in kinesthesia [54-56]. Although we do not know if refinements involving proprioceptive processing contribute to motor learning in the current study, we believe they may have facilitated increases in Gaze-Hand Distance by reducing reliance on visual feedback used to accurately execute skilled limb movements.

Task demands and environmental features are known to alter motor learning $[63,64]$. However, we did not investigate how task demands and environmental features influence the extent to which different processes are predictive of motor learning. In the current paradigm, for example, we would expect that refinements of skilled limb movements would be a greater predictor of motor learning if the demands on skilled limb movements were increased by reducing the size of the paddles or by imposing mechanical perturbations on the hands.

Although our behavioral measures probed several neural processes involved in motor learning, we did not directly investigate the underlying neural mechanisms of motor learning. Numerous studies of motor learning have explored changes in brain regions and networks related to refinements of skilled limb movement [65-67]. Other studies have investigated the brain regions and networks associated with visual search during perceptual and cognitive tasks [68-72]. However, we are unaware of any studies that have examined the extent to which brain regions and networks that underlie multiple processes are associated with motor learning.

\section{Conclusions}

Our findings indicate that motor learning may result from refinements of multiple behavioral features that are mediated by adaptations involving multiple neural processes. This knowledge may help advance post-stroke rehabilitation. Notably, most stroke survivors experience chronic difficulties performing daily motor tasks like cooking, walking, and driving [73-75], and many exhibit deficits in performing skilled limb movements [76-80], visual search [81-83], and eye-hand coordination [84, 85]. Our findings suggest that these deficits may independently alter the outcomes of rehabilitation interventions designed to target mechanisms of motor learning. Future studies that investigate the extent to which these deficits independently affect motor learning are needed to guide the development of novel neurorehabilitation interventions that can improve motor function and reduce chronic disability after stroke.

\section{Abbreviations \\ OHA: Object hit and avoid; BIC: Bayesian information criterion.}

\section{Author's contributions}

CMP, TS, KGS and TMH designed the study. CMP, KGS and ATH collected the data. CMP, TS, ACM and TMH analyzed the data. CMP drafted the initial manuscript and all authors contributed to revising the manuscript. All authors read and approved the submitted manuscript and agree to be personally accountable for the accuracy and integrity of any part of the work. All authors read and approved the final manuscript.

\section{Funding}

This study was funded by ASPIRE awards to TMH and TS, SPARC awards to CMP and ATH, and a Magellan Scholar award to KGS from the Office of Research at the University of South Carolina.

\section{Availability of data and materials}

All data used for the current study will be made available by the corresponding author on request by any qualified researcher.

\section{Ethics approval and consent to participate}

The study protocol was approved by the University of South Carolina's Institutional Review Board and all participants provided informed consent to participate.

Consent for publication

All participants provided informed consent for publication. 


\section{Competing interests}

The authors declare that they have no competing interests.Coefficient of variance $=\mathrm{SD} /$ Mean. Bold indicates measures with high correlations $(|r| \geq 0.707)$.Bold indicates measures that exhibited meaningful relationships with Targets Hit or Distractors Avoided $\left(f^{2} \geq 0.02\right)$.

\section{Author details}

${ }^{1}$ Department of Exercise Science, University of South Carolina, Columbia, SC 29208, USA. ${ }^{2}$ Department of Kinesiology, The Pennsylvania State University, University Park, PA 16802, USA. ${ }^{3}$ Department of Epidemiology and Biostatistics, University of South Carolina, Columbia, SC 29208, USA.

\section{Received: 24 September 2019 Accepted: 2 October 2020}

\section{Published online: 17 November 2020}

\section{References}

1. Shadmehr R, Smith MA, Krakauer JW. Error correction, sensory prediction, and adaptation in motor control. Annu Rev Neurosci. 2010;33:89-108.

2. Krakauer JW, Mazzoni P. Human sensorimotor learning: adaptation, skill, and beyond. Curr Opin Neurobiol. 2011;21:636-44.

3. Wolpert DM, Diedrichsen J, Flanagan JR. Principles of sensorimotor learning. Nat Rev Neurosci. 2011;12:739-51.

4. Thoroughman KA, Shadmehr R. Electromyographic correlates of learning an internal model of reaching movements. J Neurosci. 1999;19:8573-88.

5. Burdet E, Osu R, Franklin DW, Milner T, Kawato M. The central nervous system stabilizes unstable dynamics by learning optimal impedance. Nature. 2001:414:446-9.

6. Darainy M, Ostry DJ. Muscle cocontraction following dynamics learning Exp Brain Res. 2008;190:153-63.

7. Flament D, Shapiro M, KempfT, Corcos D. Time course and temporal order of changes in movement kinematics during learning of fast and accurate elbow flexions. Exp Brain Res. 1999;129:441-50.

8. Novak K, Miller L, Houk J. Features of motor performance that drive adaptation in rapid hand movements. Exp Brain Res. 2003;148:388-400.

9. van Beers R. Motor learning is optimally tuned to the properties of motor noise. Neuron. 2009;6:406-17.

10. Mosier KM, Scheidt RA, Acosta S, Mussa-Ivaldi FA. Remapping hand movements in a novel geometrical environment. J Neurophysiol. 2005;94:4362-72

11. Cohen RG, Sternad D. Variability in motor learning: relocating, channeling, and reducing noise. Exp Brain Res. 2009;193:69-83.

12. Shmuelof L, Krakauer JW, Mazzoni P. How is a motor skill learned? Change and invariance at the levels of task success and trajectory control. J Neurophysiol. 2012;108:578-94.

13. Huber ME, Kuznetsov N, Stemad D. Persistence of reduced neuromotor noise in long term motor skill learning. J Neurophysiol. 2016;116:2922-35.

14. Land MF, Mennie N, Rusted J. The roles of vision and eye movements in the control of activities of daily living. Perception. 1999;28:1311-28.

15. Land MF, Hayhoe M. In what ways do eye movements contribute to everyday activities? Vis Res. 2001;41:3559-655.

16. Mourant R, Rockwell T. Strategies of visual search by novice and experienced drivers. Hum Factors. 1972;14:325-35.

17. Vickers JN. Gaze control in putting. Perception. 1992;21:117-32.

18. Land MF, Mcleod P. From eye movements to actions: how batsmen hit the ball. Nat Neurosci. 2000;3:1340-5.

19. Williams AM, Singer RN, Frehlich SG. Quiet eye duration, expertise, and task complexity in near and far aiming tasks. J Mot Behav. 2002:34:197-207.

20. Vickers JN, Lewinski W. Performing under pressure: gaze control, decision making and shooting performance of elite and rookie police officers. Hum Mov Sci. 2012;31:101-17.

21. Shapiro KL, Raymond JE. Training of efficient oculomotor strategies enhances skill acquisition. Acta Psychol. 1989;71:217-42.

22. Harle SK, Vickers JN. Training quiet eye improves accuracy in the basketball free throw. Sport Psychol. 2001;15:289-305.

23. Causer J, Holmes PS, Williams AM. Quiet eye training in a visuomotor control task. Appl Sci. 2011;43:1042-9.
24. Wilson MR, Vine SJ, Bright E, Masters R, Defriend D, Mcgrath JS. Gaze training enhances laparoscopic technical skill acquisition and multitasking performance: a randomized, controlled study. Surg Endosc. 2011;25:3731-9.

25. Vine SJ, Chaytor RJ, McGrath JS, Masters RS, Wilson M. Gaze training improves retention and transfer of laparoscopic technical skills in novices. Surg Endosc. 2013;27:3205-13.

26. Chun MM, Jiang Y. Contextual cueing: implicit learning and memory of visual context guides spatial attention. Cogn Psychol. 1998;36:28-71.

27. van Asselen M, Sampaio J, Pina A, Castelo-Branco M. Object based implicit contextual learning: a study of eye movements. Atten Percept Psychophys. 2011;73:297-302.

28. Jones JL, Kaschak MP. Global statistical learning in a visual search task. J Exp Psychol Hum Percept Perform. 2012;38:152-60.

29. Li CL, Aivar MP, Kit DM, Tong MH, Hayhoe MM. Memory and visual search in naturalistic 2D and 3D environments. J Vis. 2016;16:9.

30. Hoppe D, Rothkopf CA. Learning rational temporal eye movement strategies. Proc Natl Acad Sci USA. 2016;113:8332-7.

31. Ma-Wyatt A, Stritzke M, Trommershäuser J. Eye-hand coordination while pointing rapidly under risk. Exp Brain Res. 2010;203:131-45.

32. Zhang H, Morvan C, Etezad-Heydari LA, Maloney L. Very slow search and reach: failure to maximize expected gain in an eye-hand coordination task. PLoS Comput Biol. 2012;8:1002718.

33. Sailer U, Flanagan JR, Johansson RS. Eye-hand coordination during learning of a novel visuomotor task. J Neurosci. 2005;25:8833-42.

34. Rand M, Stelmach $G$. Adaptation of gaze through practice in young and older adults. Neurosci Lett. 2011;492:47-51.

35. Foerster RM, Carbone E, Koesling H, Schneider WX. Saccadic eye movements in a high-speed bimanual stacking task: changes of attentional control during learning and automatization. J Vis. 2011;11:9.

36. Säfström D, Johansson RS, Flanagan JR. Gaze behavior when learning to link sequential action phases in a manual task. J Vis. 2014;14:3.

37. Scott SH. A functional taxonomy of bottom-up sensory feedback processing for motor actions. Trends Neurosci. 2016;39:512-26.

38. Brown LE, Wilson ET, Goodale MA, Gribble PL. Motor force field learning influences visual processing of target motion. J Neurosci. 2007;27:9975-83.

39. McGregor HR, Gribble PL. Changes in visual and sensory-motor restingstate functional connectivity support motor learning by observing. J Neurophysiol. 2015;114:677-88.

40. Cisek P, Kalaska JF. Neural mechanisms for interacting with a world full of action choices. Ann Rev Neurosci. 2010;33:269-98.

41. Singh T, Perry CM, Herter TM. A geometric method for computing ocular kinematics and classifying gaze events using monocular remote eye tracking in a robotic environment. J Neuroeng Rehabil. 2016;13:10.

42. Bourke TC, Lowrey CR, Dukelow SP, Bagg SD, Normal KE, Scott SH. A robot-based behavioural task to quantify impairments in rapid motor decisions and actions after stroke. J Neuroeng Rehabil. 2016;13:91.

43. Neggers SF, Bekkering $\mathrm{H}$. Ocular gaze is anchored to the target of an ongoing pointing movement. J Neurophysiol. 2000;83:639-51.

44. McDonald JH. Handbook of biological statistics. Baltimore: Sparky House Publishing; 2009

45. Holland PW, Welsch RE. Robust regression using iteratively reweighted least squares. Commun Stat Theor Meth. 1977;A6:813-28.

46. Cohen J. Statistical power analysis for the behavior sciences. 2nd ed. Hillsdale: Erlbaum; 1988.

47. Allison P. Multiple regression: a primer. Newbury Park: Pine Forge Press; 1999.

48. Ostry DJ, Gribble PL. Sensory plasticity in human motor learning. Trends Neurosci. 2016:39:114-23.

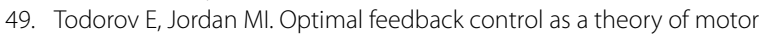
coordination. Nat Neurosci. 2002;5:1226-355.

50. Todorov E. Optimality principles in sensorimotor control. Nat Neurosci. 2004:7:907-15.

51. Neider MB, Zelinsky GJ. Scene context guides eye movements during visual search. Vis Res. 2006;46:614-21.

52. Wolfe JM, Vo ML, Evans KK, Greene MR. Visual search in scenes involves selective and nonselective pathways. Trends Cogn Sci. 2011:15:77-84

53. Wu S, Spence I. Playing shooter and driving videogames improves top-down guidance in visual search. Atten Percept Psychophys. 2013:75:673-86. 
54. Prado J, Clavagnier S, Otzenberger H, Scheiber C, Kennedy H, Perenin $\mathrm{M}$. Two cortical systems for reaching in central and peripheral vision. Neuron. 2005;48:849-58.

55. Cressman EK, Henriques DY. Sensory recalibration of hand position following visuomotor adaptation. J Neurophysiol. 2009;102:3505-18.

56. Haith A, Jackson C, Mial R, Vijayakumar S. Unifying the sensory and motor components of sensorimotor adaptation. Adv Neural Inf Process Syst. 2008;21:593-600.

57. Ostry DJ, Darainy M, Mattar AA, Wong J, Gribble PL. Somatosensory plasticity and motor learning. J Neurosci. 2010;30:5384-93.

58. Beets IA, Macé M, Meesen RL, Cuypers K, Levin O, Swinnen SP. Active versus passive training of a complex bimanual task: is prescriptive proprioceptive information sufficient for inducing motor learning? PLOS ONE. 2012;7:37687.

59. Wong JD, Kistemaker DA, Chin A, Gribble PL. Can proprioceptive training improve motor learning? J Neurophysiol. 2012;108:3313-21.

60. Bernardi NF, Darainy M, Ostry DJ. Somatosensory contribution to the initial stages of human motor learning. J Neurosci. 2015:35:14316-26.

61. Sidarta A, Vahdat S, Bernardi NF, Ostry DJ. Somatic and reinforcementbased plasticity in the initial stages of human motor learning. J Neurosci. 2016;36:11682-92.

62. Cluff T, Scott SH. Rapid feedback responses correlate with reach adaptation and properties of novel upper limb loads. J. Neurosci. 2013;33:15903-14.

63. Wright DL, Shea CH. Contextual dependencies in motor skills. Mem Cogn. 1991;19:361-70.

64. Kurtzer I, DiZio P, Lackner J. Task-dependent motor learning. Exp Brain Res. 2003;153:128-32.

65. Ghilardi M, Ghez C, Dhawan V, Moeller J, Mentis M, Nakamura T, et al. Patterns of regional brain activation associated with different forms of motor learning. Brain Res. 2000:871:127-45.

66. Frutiger SA, Strother SC, Anderson JR, Sidtis JJ, Arnold JB, Rottenberg DA. Multivariate predictive relationship between kinematic and functional activation patterns in a PET study of visuomotor learning. Neuroimage. 2000;12:515-27.

67. Muellbacher W, Ziemann U, Wissel J, Dang N, Kofler M, Facchini S, et al. Early consolidation in human primary motor cortex. Nature. 2002:415:640-4.

68. Gitelman DR, Parrish TB, Friston KJ, Mesulam MM. Functional anatomy of visual search: regional segregations within the frontal eye fields and effective connectivity of the superior colliculus. Neuroimage. 2002:15:970-82

69. Egner T, Monti JM, Trittschuh EH, Wieneke CA, Hirsch J, Mesulam MM. Neural integration of top-down spatial and feature-based information in visual search. J Neurosci. 2008;28:6141-51.

70. Weidner R, Krummenacher J, Reimann B, Müller HJ, Fink GR. Sources of top-down control in visual search. J Cogn Neurosci. 2009;21:2100-13.

71. Huang TR, Grossberg S. Cortical dynamics of contextually cued attentive visual learning and search: spatial and object evidence accumulation. Psychol Rev. 2010;117:1080-112.
72. Wei P, Yu H, Müller HJ, Pollmann S, Zhou X. Differential brain mechanisms for processing distracting information in task-relevant and irrelevant dimensions in visual search. Hum Brain Mapp. 2019;40:110-24.

73. Fisk GD, Owsley C, Pulley V. Driving after stroke: driving exposure, advice, and evaluations. Arch Phys Med Rehabil. 1997;78:1338-455.

74. Mayo NE, Wood-Dauphinee S, Ahmed S, Gordon C, Higgins J, McEwen S, et al. Disablement following stroke. Disabil Rehabil. 1999;21:258-68.

75. Mayo NE, Wood-Dauphinee S, Côté R, Durcan L, Carlton J. Activity, participation, and quality of life 6 months poststroke. Arch Phys Med Rehabil. 2002;83:1035-42

76. Levin MF. Interjoint coordination during pointing movements is disrupted in spastic hemiparesis. Brain. 1996;119:281-93.

77. Winstein CJ, Pohl PS. Effects of unilateral brain damage on the control of goal-directed hand movements. Exp Brain Res. 1995;105:163-74.

78. Beer RF, Dewald JP, Rymer WZ. Deficits in the coordination of multijoint arm movements in patients with hemiparesis: evidence for disturbed control of limb dynamics. Exp Brain Res. 2000;131:305-19.

79. Coderre AM, Zeid AA, Dukelow SP, Demmer MJ, Moore KD, Demers MJ, et al. Assessment of upper-limb sensorimotor function of subacute stroke patients using visually guided reaching. Neurorehabil Neural Repair. 2010;24:528-41.

80. Tyryshkin K, Coderre AM, Glasgow Jl, Herter TM, Bagg SD, Dukelow SP, et al. A robotic object hitting task to quantify sensorimotor impairments in participants with stroke. J Neuroeng Rehabil. 2014;11:47.

81. Zihl J, Hebel N. Patterns of oculomotor scanning in patients with unilateral posterior parietal or frontal lobe damage. Nueropsychologia. 1997:35:893-906.

82. Mapstone M, Weintraub S, Nowinski C, Kaptanoglu G, Gitelman DR, Mesulam MM. Cerebral hemispheric specialization for spatial attention: spatial distribution of search-related eye fixations in the absence of neglect. Neuropsychologia. 2003;41:1396-409.

83. Singh T, Fridriksson J, Perry CM, Tryon SC, Ross A, Fritz S, et al. A novel computational model to probe visual search deficits during motor performance. J Neurophysiol. 2017;117:79-92.

84. Rizzo JR, Fung JK, Hosseini M, Shafieesabet A, Ahdoot E, Pasculli RM, et al. Eye control deficits coupled to hand control deficits: Eye-hand incoordination in chronic cerebral injury. Front Neurol. 2017;8:330.

85. Singh T, Perry CM, Fritz S, Fridriksson J, Herter TM. Eye movements interfere with limb motor control in stroke survivors. Neurorehabil Neural Repair. 2018;32:724-34.

\section{Publisher's Note}

Springer Nature remains neutral with regard to jurisdictional claims in published maps and institutional affiliations.

Ready to submit your research? Choose BMC and benefit from

- fast, convenient online submission

- thorough peer review by experienced researchers in your field

- rapid publication on acceptance

- support for research data, including large and complex data types

- gold Open Access which fosters wider collaboration and increased citations

- maximum visibility for your research: over 100M website views per year

At BMC, research is always in progress.

Learn more biomedcentral.com/submissions 